\title{
Degenerate Mirrorless Optical Parametric Oscillator
}

\author{
Anne-Lise Viotti, Patrick Mutter, Andrius Zukauskas, Valdas Pasiskevicius and Carlota Canalias \\ Department of Applied Physics, Royal Institute of Technology, Roslagstullbacken 21, 10691, Stockholm, Sweden
}

A mirrorless optical parametric oscillator (MOPO) is a very special class of optical parametric oscillators, where the feedback for oscillation is realized by counter-propagating second-order nonlinear interaction without a need for external mirrors. This arrangement makes the MOPO a very simple, self-aligned and robust oscillator. The MOPO was first proposed theoretically in 1966 [1] however its realization was only possible using the quasiphase matching (QPM) approach due to requirement for compensation of large momentum mismatch in such interactions [2]. Realization of first-order counter-propagating parametric oscillation requires structures with periodicity in the sub- $\mu \mathrm{m}$ range, which poses substantial fabrication challenges. In addition to its self-aligned nature, MOPO offers unique spectral properties [3], whereby the spectral contents of the pump are transferred to the forward wave, while the backward wave remains narrowband. So far, only nondegenerate MOPO oscillation has been demonstrated. However, it has been theoretically proposed that a degenerate MOPO could be used to realize a high-brightness biphoton source for quantum optics applications [4].

In this work we report on the first realization of the degenerate MOPO generating counter-propagating signal and idler in the spectral band of optical communications. The MOPO was realized in periodically poled Rb-doped $\mathrm{KTiOPO}_{4}(\mathrm{PPRKTP})$ with a QPM period of $420 \mathrm{~nm}$. The structure was fabricated by the coercive field engineering technique [5]. First, the crystals were patterned using a UV laser interference lithography. Then a coercive field grating was created by periodic ion-exchange. Finally, the crystals were poled using planar electrodes. The resulting domain pattern shows excellent uniformity over the whole crystal aperture, and a duty cycle close to $50 \%$ (see Fig 1(a)). The optical surfaces of the crystals were polished at an angle to prevent Fresnel-reflection feedback into the MOPO
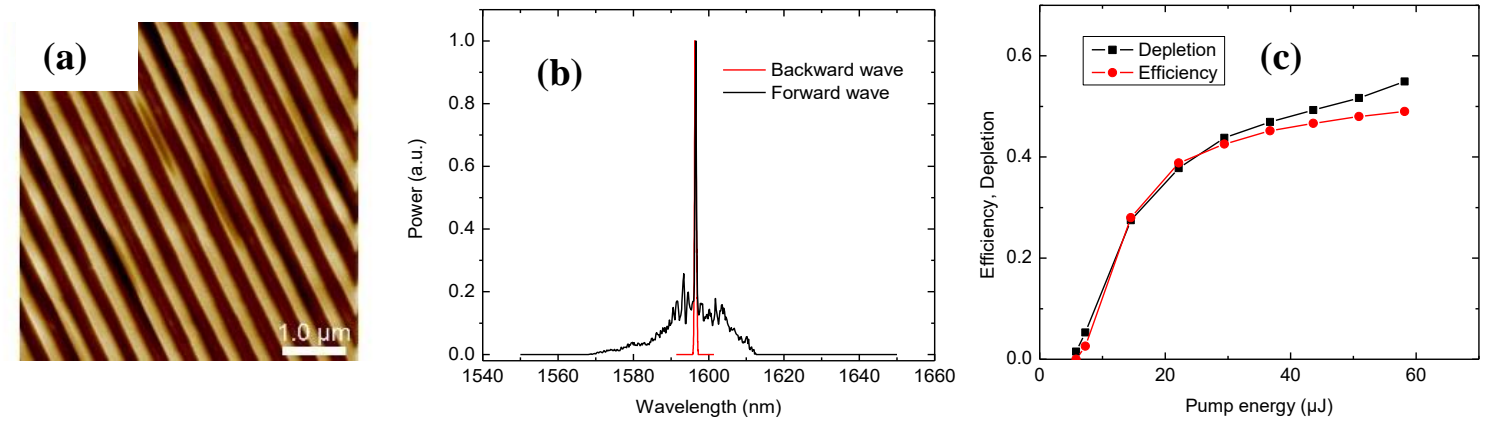

Fig. 1 (a) Atomic-force microscope image of the PPRKTP crystal. Forward- and backward-wave spectra (b), combined forward-and backward signal conversion efficiency (red) and pump depletion (black symbols) (c)

The crystals were pumped by a Ti:Sapphire regenerative amplifier delivering 294 ps-long chirped pulses at a central wavelength of $800 \mathrm{~nm}$ and a spectral bandwidth of about $10 \mathrm{~nm}$. The pump beam was focused inside the crystal to a beam radius (1/e2) of $60 \mu \mathrm{m}$. The MOPO process started at a threshold of $872 \mathrm{MW} / \mathrm{cm}^{2}$. This process generated a broadband forward wave and a narrowband backward wave, both centered around $1596.4 \mathrm{~nm}$ (Fig.1 (b)). The pump wavelength corresponding to the degenerate operation of this MOPO is $798.2 \mathrm{~nm}$. Both the forward- and backward-wave spectra have narrow $(\sim 0.5 \mathrm{~nm})$ peaks at degeneracy. The conversion efficiency in the MOPO reached $49 \%$ at the pump energy of $58 \mu \mathrm{J}$ (Fig. 1(c)). The pump depletion was determined from the measurement of the pump power transmitted through the crystal. A small discrepancy between the depletion and the measured MOPO efficiency arises due to second-harmonic ( $\mathrm{SH}$ ) generated in the backward direction by the counter-propagating degenerate MOPO waves. Indeed, the MOPO at the degeneracy point must generate forward and backward SH waves in the presence of counter-propagating degenerate signal and idler. This provides spectral coupling and locking mechanism for the MOPO at degeneracy. Therefore MOPO operation at degeneracy has similarities with doubly-resonant OPO, where the back-conversion of the signal and idler to the pump limits the maximum efficiency to $50 \%$. For the pump wavelengths outside the narrow degeneracy window of about $0.2 \mathrm{~nm}$ at $798.2 \mathrm{~nm}$, the MOPO operates in nondegenerate mode, i.e. the spectral contents of the pump is transferred to the forward wave in the MOPO, while the wavelength of the backward wave remains fixed as enforced by the momentum conservation.

\section{References}

[1] S.E. Harris, "Proposed backward wave oscillation in the infrared" Appl. Phys. Lett. 9, 114, (1966)

[2] C. Canalias, and V. Pasiskevicius, "Mirrorless Optical parametric Oscillator" Nat. Photonics 1, 459 (2007)

[3] G. Strömqvist, V. Pasiskevicius, C. Canalias and C. Montes "Coherent phase-modulation transfer in counter-propagating parametric down-conversion" Phys. Rev. A. 84, 023825 (2011)

[4] C. Chuu and S. E. Harris, "Ultrabright backward-wave biphoton source" Phys. Rev. A 83, 061803R (2011)

[5] C. Liljestrand, F. Laurell, and C. Canalias, "Periodic poling of Rb-doped $\mathrm{KTiOPO}_{4}$ " Opt. Express 24,14682, (2016) 